\title{
Soins plus - mise en place d'une intervention à trois volets visant à accroître la visibilité et la disponibilité du pharmacien dans un hôpital pédiatrique : une étude randomisée contrôlée
}

\author{
par Flaviu Mosora, Myriam Guèvremont, Gabriel Vézina, Karine Côté, Marianne Boulé, Denis Lebel, \\ Jean-François Bussières et Marie-Élaine Métras
}

J. Can. Pharm. Hosp. 2021;74(2):95-103

\section{RÉSUMÉ}

Contexte : Le rôle du pharmacien au sein de l'équipe multidisciplinaire est souvent méconnu. Diverses interventions peuvent être mises en place pour promouvoir le rôle du pharmacien en milieu hospitalier auprès des familles, des patients et des autres professionnels de la santé. Peu d'études décrivent la faisabilité et évaluent l'impact de ces interventions, particulièrement en pédiatrie.

Objectifs : Décrire l'implantation d'une intervention à trois volets visant à accrôtre la visibilité du pharmacien et de son rôle dans l'équipe traitante, pour permettre d'optimiser les soins pharmaceutiques des patients hospitalisés dans les unités de pédiatrie générale du CHU Sainte-Justine, à Montréal (Québec). Comparer la perception et la satisfaction des parents de patients hospitalisés et des soignants recevant soit des soins pharmaceutiques usuels, ou soit des soins pharmaceutiques intégrant l'intervention.

Méthode : Étude expérimentale randomisée contrôlée à simple aveugle portant sur des patients admis dans les unités de pédiatrie générale entre le 5 mars et le 8 août 2019. Outre des soins usuels, l'intervention comporte la remise d'une brochure d'information sur les services et soins pharmaceutiques, l'accès à une ligne téléphonique permettant aux familles et aux patients de prendre contact avec un résident en pharmacie pendant leur séjour à l'hôpital et jusqu'à un mois après le congé et le remplissage par le pharmacien responsable du patient d'un formulaire de congé standardisé. Un sondage de perception et de satisfaction a aussi été réalisé auprès des participants et des professionnels de la santé concernés.

Résultats : Six cent quarante et un (641) participants ont été inclus dans l'étude, 321 dans le groupe intervention et 320 dans le groupe témoin. La brochure a été remise à tous les parents du groupe intervention. Douze appels téléphoniques ont été faits au moyen d'une ligne téléphonique spéciale. Le formulaire de congé standardisé de 46,7 \% (150/321) des participants du groupe intervention a été rempli. Une majorité des parents et des patients ayant répondu au sondage $(81,2 \%, 298 / 367)$ se disent satisfaits des services et des soins pharmaceutiques reçus dans les deux groupes. Une proportion de $83,9 \%$ des participants $d u$ groupe intervention se disent satisfaits des soins et services pharmaceutiques reçus comparativement à $78,5 \%$ du groupe témoin $(p=0,18)$. De plus, $60,3 \%$ (111/184) des participants du groupe intervention disent que l'information transmise pendant l'hospitalisation concernant les médicaments leur a apporté de nouvelles connaissances, contre $48,1 \%$ (87/181) des participants du groupe témoin $(p=0,019)$. Les résultats du sondage montrent que les soignants sont en accord avec l'intervention.

Conclusion : Les trois volets de l'intervention ont été implantés dans les unités de pédiatrie sur une période de cinq mois. Cette intervention est perçue comme étant positive par les parents et les soignants concernés et les répondants ont été majoritairement satisfaits des services et des soins pharmaceutiques offerts. Mots-clés : pharmacien, ligne téléphonique, formulaire de congé standardisé, brochure d'information

\begin{abstract}
Background: The pharmacist's role within the multidisciplinary team is often poorly understood. Various interventions can be put into place to promote the role of the pharmacist in the hospital setting with families, patients, and other health care professionals. Few studies have described the feasibility and assessed the impact of such interventions, particularly in pediatrics.

Objectives: To describe the implementation of a 3-part intervention aimed at increasing the visibility of pharmacists and their role on the treatment team, with the goal of optimizing the pharmaceutical care of hospitalized patients in the general pediatric units of CHU Sainte-Justine, in Montréal, Quebec, and to compare the perceptions and satisfaction of patients' parents and of health care professionals with exposure to either usual pharmaceutical care or to pharmaceutical care incorporating the intervention.

Methods: This single-blind, randomized, controlled experimental study involved patients admitted to general pediatric units between March 5 and August 8,2019 . In addition to usual care, the intervention included delivery of an information brochure about pharmaceutical services and care, access to a telephone line (which allowed families and patients to contact a pharmacy resident during their stay in hospital and up to 1 month after discharge), and completion of a standardized discharge form by the pharmacist responsible for the patient. The participants and health professionals concerned were surveyed to determine their perceptions and level of satisfaction.
\end{abstract}

Results: A total of 641 participants were included in the study, 321 in the intervention group and 320 in the control group. The brochure was given to all parents in the intervention group. Twelve phone calls were made through the dedicated telephone line. The standardized discharge form was completed for $46.7 \%$ (150/321) of the participants in the intervention group. Most of the parents and patients who responded to the survey, in either group $(81.2 \%$, 298/367), reported satisfaction with the pharmaceutical services and care received. Of participants in the intervention group, $83.9 \%$ were satisfied with the pharmaceutical care and services received, compared with $78.5 \%$ of those in the control group $(p=0.18)$. In addition, $60.3 \%(111 / 184)$ of participants in the intervention group said that the information about medications that was provided during the hospital stay gave them new knowledge, compared with $48.1 \%$ $(87 / 181)$ of those in the control group $(p=0.019)$. The results of the survey showed that care providers were in agreement with the intervention.

Conclusions: The 3 components of the intervention were implemented in the pediatric units over a period of 5 months. The intervention was perceived as positive by the parents and care providers concerned, and the respondents were mostly satisfied with the services and pharmaceutical care offered.

Keywords: pharmacist, telephone line, standardized discharge form, information brochure 


\section{INTRODUCTION}

Les soins pharmaceutiques ont beaucoup évolué au cours des dernières décennies ${ }^{1}$. Bien que la plupart des patients hospitalisés soient en contact avec des pharmaciens, une méconnaissance du rôle de ces derniers persiste au sein de léquipe multidisciplinaire ${ }^{2-6}$. Les patients ne sont pas toujours conscients qu'il existe des pharmaciens à l'hôpital et qu'il est possible de recourir à leurs services. Dans le même ordre d'idées, le pharmacien a un rôle important en pédiatrie pour gérer des enjeux spécifiques à la population pédiatrique, tels que l'administration des médicaments ou la disponibilité de formes pharmaceutiques particulières après le congé. Plusieurs auteurs ont mis en évidence quelques interventions visant à rendre davantage visibles la présence et le rôle du pharmacien auprès des soignants et des patients ${ }^{4,7,8}$. Parmi ces interventions, on retrouve notamment la remise d'information sur le rôle du pharmacien, le bon usage des médicaments ainsi que l'utilisation d'outils contribuant à une meilleure planification du congét ${ }^{4,7}$. Dans l'optique d'une amélioration continuelle des soins pharmaceutiques offerts aux patients, nous nous sommes donc questionnés sur la possibilité de développer une intervention à visée informative et collaborative. Toutefois, il existe peu détudes évaluant la faisabilité et l'impact de ces interventions, particulièrement en pédiatrie. Nous voulons décrire l'implantation d'une intervention à trois volets et son ajout aux soins usuels dans les unités de pédiatrie générale. Notre hypothèse postule que limplantation de cette intervention est réaliste et que la satisfaction des parents concernés par l'intervention sera supérieure à celle des parents du groupe contrôle.

\section{MÉTHODE}

\section{Protocole de l'étude}

Il s'agit d'une étude descriptive, randomisée, contrôlée, à simple aveugle. Létude a été approuvée par le comité déthique de la recherche du CHU Sainte-Justine.

\section{Population à l'étude}

Létude cible tous les patients hospitalisés dans les unités de pédiatrie générale du CHU Sainte-Justine, un hôpital mère-enfant de soins tertiaires de 500 lits, situé à Montréal (Québec), entre le 5 mars et le 8 août 2019. Étant donné que l'étude portait sur une population pédiatrique, l'intervention en trois volets est davantage destinée aux parents et aux patients plus âgés (adolescents $\geq 14$ ans). Ainsi, dans ce texte, le terme " parent » inclut les patients. Les adolescents de plus de 14 ans recevaient la même information que leurs parents.

Pour être inclus dans létude, le patient devait avoir entre 0 et 20 ans et être admis ou transféré d'une autre unité au nom d'un pédiatre, d'un infectiologue, d'un neurologue, d'un généticien ou d'un immunologue dans l'une des quatre unités de pédiatrie que ciblait le projet. Il devait également avoir obtenu son congé durant la période détude. Les parents devaient détenir une adresse courriel et consentir à remplir le sondage d'évaluation des soins et services pharmaceutiques.

Les parents ne comprenant pas le français écrit, les patients réadmis durant la période de létude et ayant déjà participé à létude ou refusé d'y participer lors d'une hospitalisation antérieure, les patients ayant obtenu leur congé le même jour que leur admission, ceux transférés à une autre spécialité et ceux prenant part à une autre étude interagissant avec la présente étude étaient exclus. Les patients pour qui le contexte clinique et social était jugé inapproprié n’ont pas été recrutés.

\section{Interventions}

Les participants du groupe témoin ont reçu les soins pharmaceutiques usuels prodigués par le pharmacien responsable du patient, soit une revue quotidienne de leur dossier, la participation à la tournée médicale, une rencontre avec les patients si nécessaire et des interventions liées à leur pharmacothérapie. Les soins pharmaceutiques usuels sont donc adaptés aux patients présents aux unités et priorisés selon la charge de travail quotidienne.

Les participants du groupe intervention ont été exposés aux soins pharmaceutiques usuels ainsi quà l'intervention comportant trois volets : la remise d'une brochure d'information sur les services et soins pharmaceutiques, l'accès à une ligne téléphonique et le remplissage par le pharmacien d'un formulaire de congé standardisé.

La brochure d'information a été conçue par les pharmaciens du CHU Sainte-Justine (la brochure est disponible aux lecteurs, sur demande à l'auteure correspondante). Elle était remise aux parents du groupe intervention en format papier lors du recrutement, après la randomisation. Cette brochure de 16 pages (3250 mots) porte sur la pratique de la pharmacie hospitalière, le rôle du pharmacien hospitalier, la gestion des médicaments à l'hôpital, l'observance aux médicaments et propose quelques astuces pratiques pour la gestion et ladministration des médicaments à domicile.

La ligne téléphonique consacrée à la réalisation du protocole permettait aux parents de joindre directement un résident en pharmacie, de 8 heures à 18 heures en semaine et jusquà 28 jours après le congé. Le numéro remis aux participants était lié à un téléphone intelligent destiné à létude. Une boîte vocale recevait les appels reçus à lextérieur des heures d'ouverture et à un moment où le résident nétait pas disponible. Un délai de 24 heures ouvrables était prévu pour donner une réponse verbale aux parents. Le résident en pharmacie validait systématiquement les réponses auprès $\mathrm{du}$ pharmacien responsable du patient.

Le résident en pharmacie déposait le formulaire de congé standardisé au dossier du patient dans la section des ordonnances (Annexe 1, publiée au https://www.cjhp-online. ca/index.php/cjhp/issue/view/204). Une fois le formulaire déposé, le résident informait le pharmacien responsable du patient par courriel, par message texte ou en personne selon le moyen convenu. Le pharmacien responsable du patient 
devait remplir le formulaire afin de décrire les interventions pharmaceutiques envisagées avant le congé et faciliter la planification du congé. Le document était destiné aux autres intervenants participant aux soins du patient durant son hospitalisation, dont le médecin traitant et les infirmières présentes aux unités de soins.

\section{Objectifs}

Lobjectif primaire était de décrire l'implantation d'une intervention à trois volets visant à accroître la visibilité du pharmacien et son rôle auprès des familles et de léquipe traitante, afin doptimiser les soins pharmaceutiques.

Lobjectif secondaire était de comparer la perception et la satisfaction des parents de patients hospitalisés et des soignants relatives aux soins pharmaceutiques usuels et aux soins pharmaceutiques intégrant l'intervention.

Les données disponibles sur les caractéristiques des patients étaient recueillies auprès du parent lors du recrutement ainsi quà l'aide du dossier du patient.

Concernant la brochure d'information, nous avons documenté la remise ou non de la brochure aux patients du groupe intervention. Quant à la ligne téléphonique, le nombre d'appels reçus, le délai de réponse aux questions posées par téléphone, les heures des appels avec le parent ainsi que la durée de chaque communication ont été colligés. À la suite de la colligation de ces données, une rencontre entre tous les pharmaciens de l'équipe a eu lieu pour déterminer si l'implantation de cette intervention nalourdissait pas trop la charge de travail. Pour ce qui est du formulaire de congé standardisé, nous avons évalué le dépôt ou non du formulaire de congé dans le dossier du patient. Le journal de bord des pharmaciens, un outil préexistant de documentation quotidienne des activités pharmaceutiques, a servi à comptabiliser le nombre d'interventions faites par le pharmacien pour chaque patient de létude.

Lévaluation de la perception et de la satisfaction des parents et des soignants ayant participé à létude, liées aux services et aux soins pharmaceutiques, s'est déroulée à l'aide de sondages. Sept jours après le congé du patient, chaque parent a reçu un courriel affichant un hyperlien vers le sondage. Ceux qui navaient pas répondu recevaient entre un et trois rappels hebdomadaires. Le sondage visait à évaluer leurs connaissances du rôle du pharmacien, les soins et services qu'ils avaient reçus du Département de pharmacie, leur perception et leur satisfaction quant aux soins pharmaceutiques dont ils avaient bénéficié. Une section était aussi disponible pour les commentaires des répondants. Quant aux soignants (c.-à-d. médecins et infirmières), ils ont reçu à la fin de l'expérience une version électronique ou papier de leurs connaissances, de leur perception et de leur satisfaction relativement aux services et aux soins pharmaceutiques dispensés. Le sondage auprès des professionnels visait un objectif exploratoire servant à vérifier l'impact de ces interventions sur le personnel soignant. Une échelle de Likert à quatre choix a permis de mesurer la satisfaction $(\mathrm{TA}=$ très en accord, $\mathrm{PA}=$ partiellement en accord, $\mathrm{PD}=$ partiellement en désaccord, $\mathrm{TD}=$ totalement en désaccord) dans les deux sondages. Ceux-ci pouvaient être remplis en ligne, mais des versions papier ont été distribuées aux unités de soins pour faciliter le remplissage (Google Forms, Google LLC).

\section{Taille échantillonnale}

Un échantillon de 700 patients (soit, 350 dans chaque groupe) a été ciblé initialement. Une grande taille d'échantillon semblait adéquate pour représenter le roulement rapide des patients aux unités de pédiatrie générale et refléter les circonstances réelles d'implantation. À partir de cet échantillon de 700 patients et avec une estimation du taux de réponses des parents de $70 \%$, nous étions en mesure de détecter une différence de satisfaction de 6,5\% d'un taux de satisfaction estimé à $90 \%$ pour l'objectif secondaire. Ce taux de $90 \%$ provient d'une étude menée sur la satisfaction des patients dans une autre unité du même centre hospitalier ${ }^{9}$.

\section{Randomisation}

Les populations présentes dans chacune des quatre unités de pédiatrie générale peuvent comporter des spécificités en termes de diagnostics. Afin d'assurer une distribution adéquate de chaque population dans le groupe témoin et intervention, les investigateurs ont fait une randomisation stratifiée par unité de soins choisie. Chaque patient, dont le résident en pharmacie et le pharmacien responsable ont vérifié l'admissibilité, a reçu un numéro au hasard afin de déterminer son ordre de randomisation. Après une vérification des critères d'inclusion et d'exclusion les chercheurs ont attribué le patient à son groupe selon une randomisation par blocs de quatre, selon un ratio d'allocation 1:1, stratifiée par unité de soins (effectuée avec RANDOM.ORG, Randomness and Integrity Services Ltd, Dublin, Irlande). Ensuite, un résident en pharmacie a procédé au recrutement et à l'enrôlement dans létude. Les participants ne connaissaient pas le groupe auquel ils appartenaient, ce qui assurait le simple aveugle de cette étude.

\section{Analyses statistiques}

Les données recueillies ont été saisies dans une base de données (Access, Microsoft Corporation). Elles ont été transférées dans un chiffrier (Excel, Microsoft Corporation), puis analysées avec Excel et GraphPad (version en ligne) (GraphPad Inc).

Lévaluation de l'objectif primaire a fait appel aux seules statistiques descriptives.

Quant à l'objectif secondaire, les chercheurs ont commencé par diviser les réponses concernant la perception et la satisfaction des participants et des soignants en réponses positives (totalement en accord et partiellement en accord) et négatives (totalement en désaccord et partiellement en désaccord). Pour ce qui est du sondage visant les participants, un test du chi-carré a permis de comparer les proportions 
recueillies dans les deux groupes. Lorsque l'échantillon était inférieur à 30 participants pour une variable, les investigateurs ont eu recours au test exact de Fisher. Quand les variables du sondage étaient évaluées par une moyenne, un test de $t$ de Student a permis de comparer les moyennes entre les deux groupes. Quant au sondage pour les soignants, seules des statistiques descriptives ont été utilisées. Une valeur de $p$ inférieure à 0,05 est considérée comme statistiquement significative.

\section{RÉSULTATS}

\section{Caractéristiques des participants}

Sur les 924 patients admissibles, 283 ont été exclus en raison des critères d'exclusion ou parce qu'ils ont obtenu leur congé avant le recrutement par le résident en pharmacie. Six cent quarante et un (641) participants ont été inclus dans létude (Figure 1). Les résultats concernant les caractéristiques des participants démontrent que la randomisation ainsi que la stratification par unité de soins ont bien fonctionné et que les groupes sont comparables autant sur le plan des caractéristiques que des classes diagnostiques.

Le tableau 1 présente les caractéristiques des patients inclus dans létude.

\section{Description de l'intervention}

Les trois volets de l'intervention ont été mis en place pour les 321 participants du groupe intervention.

Après la randomisation, tous les participants du groupe intervention ( $n=321)$ ont reçu la brochure d'information remise par le résident en pharmacie lors du recrutement.

Douze appels ont été reçus par la ligne téléphonique spéciale; six de ces appels ont été pris en charge en direct et les six autres par la boîte vocale. Les parents et un pharmacien communautaire ont pris l'initiative de faire onze appels. Le tiers (4/12) des questions posées lors des appels portaient sur l'innocuité. Les autres questions concernaient les allergies, le remboursement d'un médicament, l'efficacité d'un traitement, la prise concomitante de plusieurs médicaments, le diagnostic, une dose omise ou vomie ou encore la stabilité des médicaments. Les participants pour lesquels la ligne téléphonique a été utilisée avaient en moyenne 3,2 ans et prenaient en moyenne 3,1 médicaments au congé. Le délai de réponse moyen était de 4,2 heures (médiane : 1,4 heure) entre le moment où la question était posée et le retour d'appel. Ce dernier a duré en moyenne neuf minutes.

Le formulaire de congé standardisé a été versé au dossier de tous les patients du groupe intervention mais rempli pour seulement $46,7 \%(150 / 321)$ de ces patients.

\section{Sondage auprès des parents}

Concernant lobjectif secondaire, 58,6\% (188/321) des parents du groupe intervention et 57,5\% (184/320) des parents du groupe témoin ont rempli le sondage (tableau 2). Puisque certains répondants nont pas complètement rempli le sondage, le dénominateur de chaque question est individualisé.

Une majorité des répondants $(81,2 \%, 298 / 367)$ se disent satisfaits des services et soins pharmaceutiques reçus, indépendamment du groupe dans lequel ils étaient intégrés. Dans le groupe intervention, 83,9 \% se disent partiellement ou totalement satisfaits comparativement à $78,5 \%$ dans le groupe témoin, mais cette différence nétait pas statistiquement significative $(p=0,18)$.

Des 57 répondants $(15,3 \%, 57 / 372)$ disant avoir reçu les services d'un pharmacien pendant l'hospitalisation, 49 $(86 \%, 49 / 57)$ se disent totalement satisfaits, six $(10,5 \%, 6 / 57)$ se disent partiellement satisfaits de leurs échanges avec le pharmacien. Dans le groupe intervention, 96,9\% (31/32) se

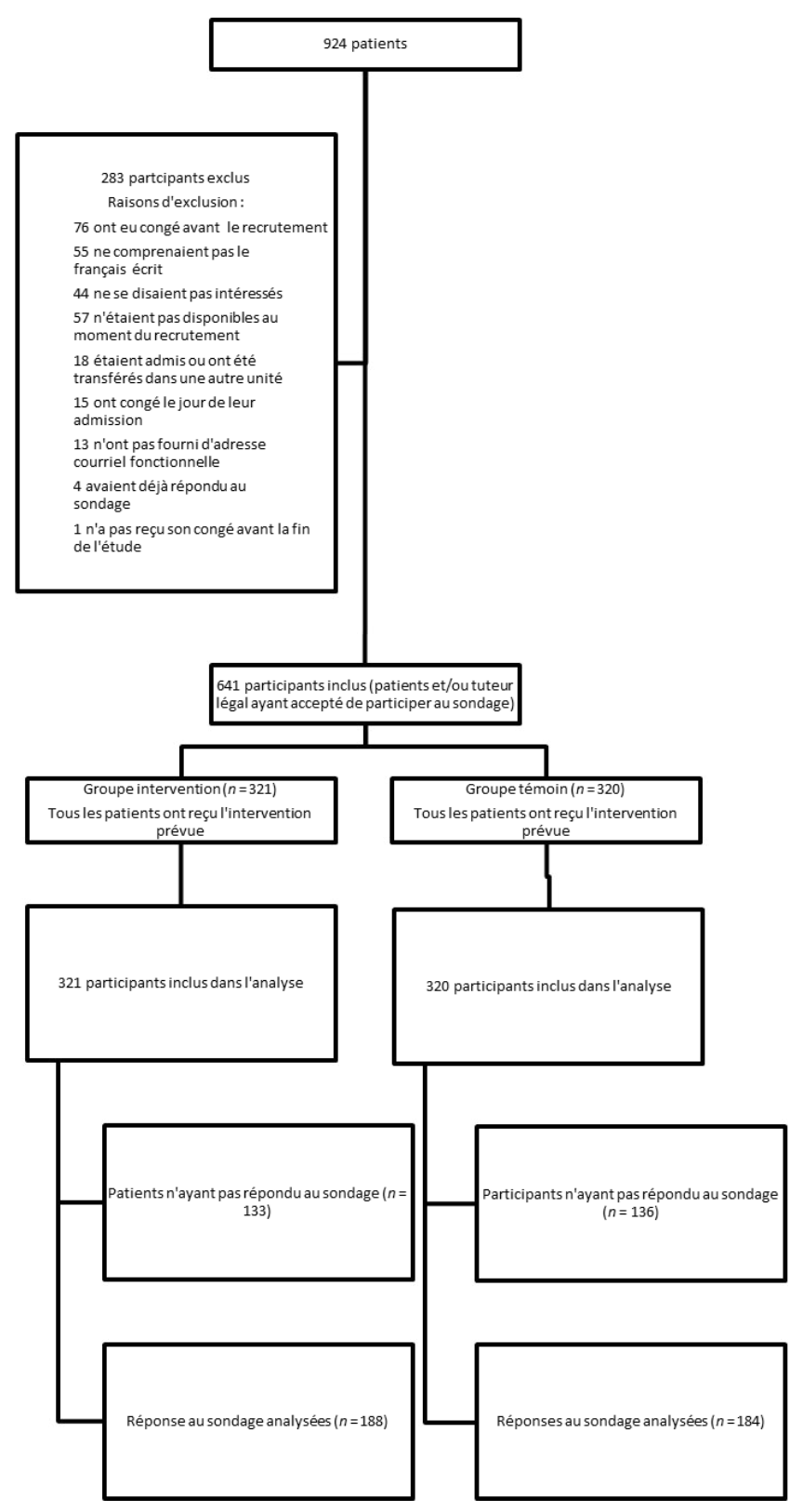

FIGURE 1. Diagramme de flux des participants. 
disent partiellement ou totalement satisfaits comparativement à $96 \%(24 / 25)$ du groupe témoin $(p=0,86)$.

À la suite de la remise de la brochure, une majorité (88\%, 161/183) des répondants sont partiellement ou totalement en accord avec le fait que le rôle du pharmacien est méconnu et qu'on devrait mieux informer les patients, contre $83,2 \%$ $(149 / 179)$ du groupe témoin $(p=0,20)$. Les parents ayant reçu la brochure ne démontraient pas une meilleure connaissance des rôles du pharmacien clinique ( 4,6 rôles mentionnés contre 4,3 dans le groupe témoin) $(p=0,10)$. Cependant, $60,3 \%(111 / 184)$ des participants du groupe intervention disent être partiellement ou totalement d'accord que l'information transmise pendant l'hospitalisation concernant les médicaments leur a apporté de nouvelles connaissances, contre 48,1\%(87/181) de ceux du groupe témoin $(p=0,019)$, ce qui résulte en une différence statistiquement significative. Les commentaires des répondants reflètent aussi le fait que les parents sont mal informés de la disponibilité du pharmacien à l'unité de pédiatrie et qu'ils auraient aimé avoir un contact avec le pharmacien durant l'hospitalisation de leur enfant.

Il existe des incohérences dans les résultats du sondage. Une proportion de 75,7 \% (78/103) des participants du groupe intervention qui ont affirmé avoir reçu de l'information de la part du pharmacien ont dit avoir reçu la brochure.
Étonnamment, 27,1 \% (13/48) des répondants du groupe témoin ont aussi répondu avoir reçu la brochure. Par ailleurs, $47,6 \%(151 / 317)$ des participants ont répondu avoir reçu de l'information du pharmacien, mais 70,2 \% (106/151) ont également répondu n'avoir eu aucun contact avecle pharmacien pendant leur séjour, ce qui semble contradictoire. Un seul des participants ayant utilisé les services de la ligne téléphonique a répondu avoir utilisé cet outil de communication. De plus, les trois autres répondants qui ont dit l'avoir utilisé n'ont aucun appel documenté associé à leur numéro de participant.

\section{Sondage auprès des soignants}

En ce qui concerne l'objectif secondaire, 23 sondages ont été remplis à la suite de l'intervention. Le tableau 3 présente la perception et la satisfaction des soignants vis-à-vis des soins et services pharmaceutiques offerts dans les deux groupes.

Les résultats du sondage montrent que les soignants sont en accord avec l'intervention. Une grande majorité des répondants ont répondu être totalement ou partiellement en accord avec le fait que les trois volets de l'intervention sont une excellente idée (tableau 3 ). Une proportion de $31,8 \%(7 / 22)$ des professionnels ayant répondu au sondage disent avoir remarqué qu'à la suite à l'intervention, les notes pharmaceutiques relatives au congé étaient plus explicites.

TABLEAU 1. Caractéristiques des patients inclus dans l'étude

\begin{tabular}{|c|c|c|}
\hline Caractéristiques & $\begin{array}{l}\text { Groupe intervention } \\
\qquad(n=321)\end{array}$ & $\begin{array}{l}\text { Groupe témoin } \\
\quad(n=320)\end{array}$ \\
\hline \multicolumn{3}{|l|}{ Âge au moment du recrutement (ans) } \\
\hline Moyenne \pm écart-type & $4,0 \pm 4,9$ & $4,4 \pm 5,1$ \\
\hline Médiane & 1,7 & 1,9 \\
\hline Assurance médicament privée, $n(\%)$ & $220(68,5)$ & $228 \quad(71,3)$ \\
\hline $\begin{array}{l}\text { Nombre moyen de médicaments par patient à l'admission, y compris les } \\
\text { médicaments au besoin (moyenne } \pm \text { écart-type) }\end{array}$ & $1,7 \pm 2,0$ & $1,6 \pm 2,0$ \\
\hline $\begin{array}{l}\text { Nombre moyen de médicaments prescrits au départ du CHU Sainte-Justine } \\
\text { (moyenne } \pm \text { écart-type) }\end{array}$ & $2,5 \pm 2,2$ & $2,5 \pm 2,3$ \\
\hline \multicolumn{3}{|l|}{ Classes diagnostiques, $n(\%)$} \\
\hline Infectiologie & $203 \quad(63,2)$ & $192(60,0)$ \\
\hline Gastroentérologie & $45 \quad(14,0)$ & $38 \quad(11,9)$ \\
\hline Neurologie & $21 \quad(6,5)$ & $23 \quad(7,2)$ \\
\hline Pneumologie & $20 \quad(6,2)$ & $15 \quad(4,7)$ \\
\hline Rhumatologie / immunologie & $11 \quad(3,4)$ & $13 \quad(4,1)$ \\
\hline Psychiatrie & $4 \quad(1,2)$ & $6 \quad(1,9)$ \\
\hline Génétique & $3 \quad(0,9)$ & $4 \quad(1,3)$ \\
\hline Hématologie & $2 \quad(0,6)$ & $8 \quad(2,5)$ \\
\hline Autres & $12 \quad(3,7)$ & $21 \quad(6,6)$ \\
\hline Nombre moyen de jours d'hospitalisation (moyenne \pm écart-type) & $5,0 \pm 6,2$ & $4,6 \pm 5,0$ \\
\hline $\begin{array}{l}\text { Nombre moyen d'hospitalisations au CHU Sainte-Justine dans la dernière } \\
\text { année (moyenne } \pm \text { écart-type) }\end{array}$ & $0,7 \pm 1,7$ & $0,5 \pm 1,3$ \\
\hline $\begin{array}{l}\text { Nombre moyen d'interventions par patient, que les pharmaciens de l'équipe } \\
\text { de pédiatrie ont documentées dans le journal de bord (moyenne } \pm \text { écart-type) }\end{array}$ & $2,4 \pm 2,5$ & $2,3 \pm 2,6$ \\
\hline
\end{tabular}


TABLEAU 2. Perception et satisfaction des parents vis-à-vis des soins et services pharmaceutiques offerts dans les deux groupes

\begin{tabular}{|c|c|c|c|}
\hline Issues & $\begin{array}{l}\text { Groupe } \\
\text { intervention }\end{array}$ & $\begin{array}{l}\text { Groupe } \\
\text { témoin }\end{array}$ & $\begin{array}{l}\text { Valeur } \\
\text { de } p\end{array}$ \\
\hline Taux de réponse au sondage, $n / N(\%)$ & $188 / 321(58,6)$ & $184 / 320(57,5)$ & 0,78 \\
\hline $\begin{array}{l}\text { Satisfaction globale des parents vis-à-vis des services et soins } \\
\text { pharmaceutiques reçus, } n / N(\%) \text { satisfaits }\end{array}$ & $156 / 186(83,9)$ & $142 / 181(78,5)$ & 0,18 \\
\hline \multicolumn{4}{|l|}{ Impact de la brochure d'information } \\
\hline $\begin{array}{l}\text { Nombre de rôles du pharmacien mentionnés par les répondants sur } \\
\text { les sept rôles proposés (moyenne } \pm \text { écart-type) }\end{array}$ & $4,6 \pm 1,9$ & $4,3 \pm 1,8$ & 0,10 \\
\hline $\begin{array}{l}\text { Répondants en accord avec l'énoncé : « L'information transmise } \\
\text { concernant les médicaments m'a apporté de nouvelles } \\
\text { connaissances », n/N (\%) }\end{array}$ & $111 / 184(60,3)$ & $87 / 181(48,1)$ & 0,019 \\
\hline $\begin{array}{l}\text { Satisfaction des parents vis-à-vis des échanges avec le pharmacien, } \\
\text { nIN (\%) satisfaits }\end{array}$ & $31 / 32(96,9)$ & $24 / 25(96,0)$ & 0,86 \\
\hline $\begin{array}{l}\text { Proportion des répondants disant avoir tenté d'entrer en contact avec le } \\
\text { pharmacien pendant leur séjour ou celui de leur enfant, } n / N(\%)\end{array}$ & $17 / 188(9,0)$ & $9 / 184(4,9)$ & 0,12 \\
\hline \multicolumn{4}{|l|}{$\begin{array}{l}\text { Nombre (\%) de parents ayant tenté d'entrer en contact avec le pharmacien } \\
\text { pendant leur séjour ou celui de leur enfant }\end{array}$} \\
\hline En le rencontrant directement & $5 / 17(29,4)$ & $6 / 9(66,6)$ & 0,10 \\
\hline En demandant au personnel médical & $11 / 17(64,7)$ & $5 / 9(55,6)$ & 0,69 \\
\hline Par la ligne téléphonique & $3 / 17(17,6)$ & $1 / 9(11,1)$ & $>0,99$ \\
\hline En posant la question au résident en pharmacie lors du recrutement & $1 / 17(5,9)$ & $0 / 0(0,0)$ & $>0,99$ \\
\hline $\begin{array}{l}\text { Nombre }(\%) \text { des répondants disant avoir contacté le pharmacien pendant } \\
\text { leur séjour ou celui de leur enfant }\end{array}$ & $32 / 188(17,0)$ & $25 / 184(13,6)$ & 0,36 \\
\hline $\begin{array}{l}\text { Nombre de fois où le répondant dit avoir joint le pharmacien pendant son } \\
\text { séjour ou celui de son enfant (moyenne } \pm \text { écart-type) }\end{array}$ & $1,4 \pm 0,6$ & $1,5 \pm 0,7$ & 0,57 \\
\hline
\end{tabular}

\section{DISCUSSION}

Pour ce qui est de l'objectif principal, cette étude a permis de décrire la mise en place de l'intervention concernant chacun des trois volets.

Quant à l'objectif secondaire, comme le degré de satisfaction des parents à l'endroit des soins et services pharmaceutiques usuels était déjà élevé, il n’a pas été possible de démontrer une augmentation de la satisfaction après la mise en place de notre intervention dans le groupe intervention par rapport au groupe témoin. Les soignants ayant participé aux interventions sont quant à eux en accord avec la mise en place de ces trois volets.

\section{Brochure d'information}

Notre étude révèle un intérêt réel pour la remise aux parents d'une brochure d'information sur les services et soins pharmaceutiques. Tous les patients du groupe intervention ont reçu la brochure. Ils ont été plus nombreux que ceux du groupe témoin à répondre qu'ils ont acquis de nouvelles connaissances sur les médicaments. Les commentaires recueillis lors du sondage reflètent également que les parents connaissaient peu le rôle du pharmacien et que plusieurs ignoraient sa présence dans l'hôpital et dans l'équipe de soins. Bajwa et collab. ont également remis à des parents une brochure décrivant le rôle du pharmacien dans une unité de soins intensifs néonataux pour augmenter la visibilité de ce professionnel de la santé ${ }^{5}$. La distribution de la brochure na pas permis d'augmenter le nombre de familles se souvenant d'avoir rencontré le pharmacien pendant leur séjour, mais les résultats indiquent que les patients aimeraient le rencontrer pendant leur hospitalisation ${ }^{5}$. Plusieurs répondants à notre sondage ont aussi manifesté de l'intérêt à rencontrer le pharmacien et pensent que les parents devraient être mieux informés de sa présence. Notre brochure a été produite en collaboration avec les éditions de l'Hôpital. Elle est donc disponible dans une section spéciale de leur site internet et le grand public peut y accéder en ligne. Certains pharmaciens travaillant dans des milieux externes ont même commencé à diffuser la brochure pour les conseils quelle contient et ils nous ont transmis des commentaires positifs.

À la lumière de ces résultats, la brochure d'information sera disponible à léchelle de létablissement. Il est probable quelle nécessite des adaptations en fonction de certaines patientèles (p. ex. oncologie, obstétrique-gynécologie, néonatologie). Des discussions sont en cours afin de déterminer le mode 
TABLEAU 3. Perception et satisfaction des soignants vis-à-vis des soins et services pharmaceutiques offerts dans les deux groupes

\begin{tabular}{|c|c|c|}
\hline Variables & Post-e & osition \\
\hline \multicolumn{3}{|l|}{ Profession des répondants } \\
\hline Médecins & $8 / 23$ & $(34,8)$ \\
\hline Infirmières & $13 / 23$ & $(56,5)$ \\
\hline Autre & $2 / 23$ & $(8,7)$ \\
\hline $\begin{array}{l}\text { Brochure : La remise systématique d'un guide d'accueil par les services et soins pharmaceutiques aux patients } \\
\text { hospitalisés est une excellente idée [en accord] }\end{array}$ & $20 / 23$ & $(87,0)$ \\
\hline $\begin{array}{l}\text { Ligne téléphonique : La remise d'un numéro de téléphone permettant aux patients de joindre directement le } \\
\text { pharmacien durant le séjour hospitalier est une excellente idée [en accord] }\end{array}$ & $21 / 23$ & $(91,3)$ \\
\hline $\begin{array}{l}\text { Formulaire de congé standardisé : La mention explicite des attentes du pharmacien à l'égard du congé du patient } \\
\text { est une excellente idée [en accord] }\end{array}$ & $22 / 23$ & $(95,7)$ \\
\hline Réponse à l'énoncé : J'ai remarqué la présence de notes plus explicites des pharmaciens concernant le congé [Oui] & $7 / 22$ & $(31,8)$ \\
\hline
\end{tabular}

optimal de diffusion qui tienne compte de nos préoccupations environnementales (possibilité d'inscrire le code QR sur une carte d'affaires ou documentation remise aux patients).

\section{Ligne téléphonique}

En ce qui concerne la ligne téléphonique, le nombre d’appels reçus était limité, ce qui laisse penser que cette mesure ne devrait pas affecter de façon importante la charge de travail des pharmaciens. En effet, il semble réaliste d'intégrer le temps nécessaire à la gestion des appels dans l'horaire du pharmacien, puisqu'on sattend à moins de deux appels par semaine et que le nombre d'appels pourrait même augmenter légèrement. Toutefois, le faible nombre d'appels (taux d'utilisation de la ligne téléphonique : 3,1\%) peut être lié au fait que le recrutement était effectué par un résident en pharmacie ne participant pas aux soins plutôt que par le pharmacien lui-même responsable du patient. De plus, les parents ont la possibilité de prendre contact avec un pharmacien de différentes façons lors de l'hospitalisation. En effet, parmi les parents qui ont tenté de contacter un pharmacien, certains l'ont fait en le rencontrant directement ou en demandant à l'équipe médicale de le rencontrer. La ligne téléphonique peut donc avoir servi à répondre à quelques questions supplémentaires, surtout après le congé du patient. Au total, la ligne téléphonique a permis de répondre à 12 questions. La plupart des appelants ont téléphoné après le congé (11/12). En l'absence de ligne téléphonique, le pharmacien communautaire du patient aurait probablement pu répondre à la majorité des questions. Cependant, la connaissance du dossier hospitalier est un avantage pour répondre aux questions, comme en témoignent les résultats de Badiani et collab. qui ont observé que laccès aux données locales, y compris au dossier du patient, aux procédures locales ou à l'accès à un professionnel connaissant le dossier du patient, était nécessaire dans $74,8 \%$ des appels ${ }^{10}$. Des sondages évaluant lexpérience avec des lignes téléphoniques destinées aux patients et prises en charge par les pharmaciens d'hôpitaux démontrent que cet outil augmente la satisfaction des patients, les rassure et permet la résolution de problèmes ${ }^{10-12}$. Quant au délai de réponse, il est probablement surestimé en raison de la nécessité de valider les réponses des résidents auprès du pharmacien responsable du patient.

À la lumière de ces résultats, la ligne téléphonique sera élargie à d'autres unités de soins au moyen d'appareils téléphoniques attribués à certaines équipes de pharmaciens, qui serviront également aux appels entre soignants. Les pharmaciens pourront choisir de donner le numéro de téléphone à des parents ciblés.

\section{Formulaire de congé standardisé}

Les pharmaciens ayant participé à notre étude ont soulevé le fait que le formulaire nétait pas pertinent pour tous les patients et que son remplissage systématique représentait une charge de travail importante sans retombées favorables, ce qui pourrait expliquer le taux de remplissage de 47,6\% du formulaire de congé standardisé. Ainsi, le formulaire était déposé au dossier, mais nétait pas toujours rempli. En effet, cétait le pharmacien responsable du patient qui remplissait normalement le formulaire de congé standardisé lorsqu'une ou des interventions étaient nécessaires, par exemple avant un congé. Quand il devait ajouter un nouveau médicament sous forme de préparation magistrale ou que le médicament nécessitait une mise au courant spécifique et plus complexe (p. ex : antibiotique intraveineux à domicile) ou encore qu'un médicament nécessitait des démarches supplémentaires auprès des assurances, sa charge de travail s’alourdissait. Étant donné que la majorité de la population pédiatrique a un dossier pharmacologique relativement simple, le formulaire de congé standardisé nétait nécessaire que pour un nombre restreint de patients. Un pharmacien désirant absolument voir un patient avant son congé devait continuer d'utiliser d'autres approches pour le faire, comme un avis verbal à l'infirmière et au médecin traitant. 
Plusieurs études mettent en évidence l'utilité d'une liste de contrôle multidisciplinaire à dresser avant le congé d'un patient adulte ${ }^{13-18}$. Voirol et collab. ont évalué l'effet d'un programme de congé standardisé par le pharmacien en pédiatrie et ont noté un impact positif sur le temps nécessaire à l’obtention des médicaments après le congé et une satisfaction accrue des parents ${ }^{8}$. Au Québec, le ministère de la Santé et des Services sociaux considère que le fait d'instaurer une fiche de transfert de soins comme formulaire de congé standardisé est une bonne pratique ${ }^{19}$. De façon générale, ces études montrent qu'un tel formulaire peut contribuer à une optimisation du traitement, à une meilleure communication entre les professionnels de la santé et à un meilleur suivi.

À la lumière de ces résultats, le formulaire de congé standardisé sera revu et amélioré. Les résultats démontrent qu'il est possible d'intégrer le formulaire de congé standardisé à chacun des dossiers, mais le taux de remplissage inférieur à $50 \%$ indique qu'il faut réévaluer la façon de procéder. Des critères de sélection des patients visés par le formulaire seront également déterminés. Avec le déploiement progressif du prescripteur électronique, on pense pouvoir intégrer les éléments clés de ce formulaire à loutil électronique avec une possibilité de notification automatique envoyée au médecin traitant.

\section{Satisfaction}

Les résultats $\mathrm{du}$ sondage montrent que les familles sont satisfaites des services et soins pharmaceutiques reçus. Il était difficile de voir une augmentation du taux de satisfaction puisque celle-ci était déjà élevée avec les soins usuels. Il est également difficile d'évaluer l'effet isolé de chacun des volets sur la satisfaction, puisqu'ils ont été implantés de façon simultanée. L'évaluation sera poursuivie afin de s'assurer d'une mise en place optimale. Entre autres, il est prévu d'ajouter une question au sondage général de l'hôpital remis aux parents avant le congé afin de connaître la satisfaction par rapport aux services et soins pharmaceutiques qu'ils ont reçus.

L'intégration des trois volets de l'intervention visant à accroître la visibilité du pharmacien et de son rôle dans l'équipe traitante, afin d'optimiser les soins pharmaceutiques, nous semble réaliste et est peu coûteuse. Intégrée au travail courant des pharmaciens, le coût de l'intervention se limite à l'impression de la brochure d'information ( $0,65 \$$ par copie) et à l'achat de quelques téléphones pour létablissement (2000 \$ de frais par année).

Cette étude comporte plusieurs forces. Les résultats reposent sur un échantillon randomisé entre deux groupes comparables. Les répondants et non-répondants au sondage avaient également des caractéristiques similaires en ce qui concerne l'âge, le nombre de médicaments et la durée de l'hospitalisation. Le choix d'un sondage à remplir en ligne de façon anonyme a permis d'obtenir un grand nombre de réponses et de limiter les risques de biais de désirabilité. Le taux de réponses (58\%) est appréciable et supérieur à celui mentionné dans la littérature scientifique ${ }^{20}$. De plus, les participants pouvaient ajouter des commentaires, ce qui a permis de recueillir de l'information supplémentaire et de mettre en contexte les résultats du sondage.

Cette étude comporte également des limites. Les réponses des parents renferment des incohérences, notamment quant au fait d'avoir reçu ou non la brochure et d'avoir fait usage ou non de la ligne téléphonique. Ces incohérences questionnent la validité des réponses, dont celles entourant la satisfaction des parents. Il est possible que des parents aient confondu la brochure d'information avec d'autres informations écrites remises lors de l'hospitalisation ou avec des fiches d'information sur les médicaments parfois remises au congé. De plus, les parents rencontrent de nombreux professionnels de la santé et ils reçoivent beaucoup d'information lors du séjour à l'hôpital, ce qui pourrait causer de la confusion et des incohérences notées dans le sondage des participants. Il est aussi possible que les parents aient rencontré des pharmaciens à d'autres unités de soins avant leur transfert aux unités de pédiatrie générale. Il est aussi possible de contacter un pharmacien de l'établissement pour lui poser des questions sans passer par la ligne téléphonique du projet. Certains répondants semblent également avoir mal compris la différence entre totalement en désaccord et non applicable. Le sondage est rempli en ligne postérieurement à l'hospitalisation et jusqu'à 28 jours après le congé, un biais de mémoire est donc probable. La littérature scientifique rapporte également ce biais. Sidhu et collab. ont évalué la validité d'un sondage demandant à des patients hospitalisés s'ils se souvenaient d'avoir rencontré un pharmacien ${ }^{6}$. Les auteurs notent que la réponse était peu fiable. Ainsi, 36,4 \% des répondants disant avoir vu le pharmacien avaient une note au dossier à cet effet et $37,2 \%$ des répondants pour lesquels il y a avait une note du pharmacien au dossier ont répondu ne pas l'avoir vu' ${ }^{6}$. Il est également possible que les mots utilisés dans les questions du sondage aient été mal compris (p. ex. confusion possible entre l'expression "résident en pharmacie » et le terme "pharmacien» ou encore entre " brochure sur les services et soins pharmaceutiques " et " feuillet d'information sur les médicaments »). Finalement, certains auteurs de cet article sont des pharmaciens exerçant au sein des unités de pédiatrie évaluées dans le cadre de cette étude et un biais de performance n'est pas exclu.

\section{CONCLUSION}

Cette étude a permis d'implanter en établissement de santé une intervention pharmaceutique à trois volets (c.-à-d. brochure d'information, ligne téléphonique, formulaire de congé standardisé) durant une période de cinq mois. Bien que des modifications seront nécessaires pour poursuivre l'implantation de ce type d'intervention, les parents et les soignants concernés ont perçu ce projet comme étant positif. Il semble essentiel que le pharmacien détablissement 
soit davantage visible auprès des patientèles pour optimiser les retombées de sa collaboration. Bien qu'il ne soit pas nécessaire ni réaliste qu'un pharmacien voie tous les patients hospitalisés, compte tenu des ressources disponibles et des besoins pharmaceutiques de ces patients, il est légitime de penser qu'une meilleure visibilité puisse contribuer à de meilleurs soins. Avec l'ajout de nouvelles activités réservées, confiées au pharmacien à l'échelle du Canada au cours des dernières années, les patients peuvent compter sur le rôle croissant des pharmaciens hospitaliers.

\section{Références}

1. Bussières JF, Tanguay C, Bonnici A. Perspective québécoise et canadienne de la pratique pharmaceutique en établissement de santé pour 2016-2017. Pharmactuel. 2018;51(2):105-42.

2. Bolduc B. Le rôle méconnu des pharmaciens [blogue]. Ordre des pharmaciens du Québec; 2019 [page consultée le 22 octobre 2019]. [en ligne] : http://blogue.opq.org/2019/03/11/le-role-meconnu-des-pharmaciens/

3. Hospital pharmacy in Canada 2013/2014 report. Hospital Pharmacy in Canada Editorial Board; 2015 [page consultée le 22 octobre 2019]. [en ligne] : https://www.cshp.ca/sites/default/files/HPC\%20Survey/ FULL-2015.pdf

4. Millin B. La sensibilisation à la pharmacie : une action de tous les jours. Can J Hosp Pharm. 2015;68(2):181.

5. Bajwa R, Kendrick JG, Carr R. The invisible white coat: awareness of pharmacists in a neonatal intensive care unit. Can J Hosp Pharm. 2014; 67(4):292-7.

6. Sidhu VK, Bresee L, Kemp K, Koshman S, Pereira T, Neilson S. Accuracy of inpatient recall of interaction with a pharmacist: a validation study from 2 acute care teaching hospitals. J Patient Exp. 2019;6(1):62-7.

7. Recommandations sur la pratique de la pharmacie en établissement de santé. Axe 1 : Soins pharmaceutiques. Association des pharmaciens des établissements de santé du Québec; 2018 [page consultée le 22 octobre 2019]. [en ligne] : https://www.apesquebec.org/sites/default/files/ publications/ouvrages_specialises/20180424_publications_pubspec_ projet1.pdf

8. Voirol P, Kayser S, Chang C, Chang Q, Youmans S. Impact of pharmacists' interventions on the pediatric discharge medication process. Ann Pharmacother. 2004;38(10):1597-602.

9. Marino-Martinez C, Prémonville L, Morin C, Ferreira E, Lebel D, Bussières JF. Évaluation des soins pharmaceutiques en obstétriquegynécologie: satisfaction des patients [affiche]. Congrès de l'Association des pharmaciens des établissements de santé du Québec; 14 avril 2016; Trois-Rivières (Québec).

10. Badiani A, Wills S, Owen S, Parker J, Hall J. Impact of a medicines helpline for patients. Eur J Hosp Pharm. 2017;24(4):196-9.

11. Olofinjana O, Connolly A, Taylor D. Outcomes of information provision to callers to a psychiatric medication helpline. Psychiatr Bull.2009; 33(10):364-7.

12. Williams M, Jordan A, Scott J, Jones MD. Operating a patient medicines helpline: a survey study exploring current practice in England using the RE-AIM evaluation framework. BMC Health Serv Res. 2018; 18(1):868.

13. Kuusisto A, Joensuu A, Nevalainen M, Pakkanen T, Ranne P, Puustinen J. Standardizing key issues from hospital through an electronic multi-professional discharge checklist to ensure continuity of care. Stud Health Technol Inform. 2019;264:664-8.

14. Legallois D, Chaufourier L, Blanchart K, Parienti JJ, Belin A, Milliez P, et al. Improving quality of care in patients with decompensated acute heart failure using a discharge checklist. Arch Cardiovasc Dis. 2019; 112(8-9):494-501.
15. Ridgeway JA. Interdisciplinary educational checklist for allogeneic stem cell transplant patients. J Adv Pract Oncol. 2018;9(6):646-54.

16. Lee R, Malfair S, Schneider J, Sidhu S, Lang C, Bredenkamp N, et al. Evaluation of pharmacist intervention on discharge medication reconciliation. Can J Hosp Pharm. 2019;72(2):111-8.

17. Prince M, Allen D, Chittenden S, Misuraca J, Hockenberry MJ. Improving transitional care: the role of handoffs and discharge checklists in hematologic malignancies. Clin J Oncol Nurs. 2019;23(1):36-42.

18. Duffy AP, Bemben NM, Li J, Trovato J. Facilitating home hospice transitions of care in oncology: evaluation of clinical pharmacists' interventions, hospice program satisfaction, and patient representation rates. Am J Hosp Palliat Care. 2018;35(9):1181-7.

19. Provencher V. Un parcours de soins et de services fluide: des transitions efficaces [présentation de diapositives]. Ministère de la Santé et des Services sociaux [Québec]; 2017 [page consultée le 22 octobre 2019]. [en ligne] : http://www.msss.gouv.qc.ca/professionnels/documents/ forum-sad/6_Bloc_3_Veronique_Provencher.pdf?fbclid=IwAR06kO roY9Y5arALAnrJvQrjXC9m7Ypp_Oj_IzRu7--APGSP82X-lRtTwE4

20. Bartel Sheehan K. E-mail survey response rates: a review. J ComputerMediated Commun. 2001;6(2):JCMC621. doi: 10.1111/j.1083-6101.2001 tb00117.x

Flaviu Mosora, Pharm. D., est candidat à la maîtrise en pharmacothérapie avancée, Unité de recherche en pratique pharmaceutique, CHU Sainte-Justine, Montréal (Québec).

Myriam Guèvremont, Pharm. D., est candidate à la maîtrise en pharmacothérapie avancée, Unité de recherche en pratique pharmaceutique, CHU Sainte-Justine, Montréal (Québec).

Gabriel Vézina, Pharm. D., est candidat à la maîtrise en pharmacothérapie avancée, Unité de recherche en pratique pharmaceutique, CHU Sainte-Justine, Montréal (Québec).

Karine Côté, Pharm. D., est candidate à la maîtrise en pharmacothérapie avancée, Unité de recherche en pratique pharmaceutique, CHU Sainte-Justine, Montréal (Québec).

Marianne Boulé, Pharm. D., M. Sc., est pharmacienne au Département de pharmacie et Unité de recherche en pratique pharmaceutique, CHU Sainte-Justine, Montréal (Québec).

Denis Lebel, M. Sc., FCSHP, est pharmacien et chef adjoint, soins, enseignement et recherche, au Département de pharmacie et Unité de recherche en pratique pharmaceutique, CHU Sainte-Justine, Montréal (Québec).

Jean-François Bussières, B. Pharm., M. Sc., MBA, FCSHP, FOPQ, est pharmacien et chef au Département de pharmacie et Unité de recherche en pratique pharmaceutique, CHU Sainte-Justine, et professeur titulaire de clinique, Faculté de pharmacie, Université de Montréal, Montréal (Québec).

Marie-Élaine Métras, Pharm. D., M. Sc., est pharmacienne au Département de pharmacie et Unité de recherche en pratique pharmaceutique, CHU Sainte-Justine, Montréal (Québec).

Conflits d'intérêts : Aucune déclaration.

Adresse de correspondance:

Marie-Élaine Métras

CHU Sainte-Justine

3175, chemin de la Côte-Sainte-Catherine

Montréal QC H3T 1C5

Courriel : marie-elaine.metras.hsj@ssss.gouv.qc.ca

Financement : Les frais associés au matériel (téléphones cellulaires, impression des dépliants, des notes de congé standardisées et des cartes d'affaires) nécessaire à la réalisation de cette étude ont été assumés par le CHU Sainte-Justine.

Remerciements : Nous tenons à remercier tous les pharmaciens qui ont participé à la prestation de soins pharmaceutiques lors de la réalisation de ce projet et tous les soignants ayant contribué à son succès. 